CORRESPONDENCE

\section{Acanthamoeba keratitis: risk factors and outcome}

EDrToR,-The paper by Illingworth and colleagues ${ }^{1}$ was useful since it identified: firstly, that the occurrence of Acanthamoeba keratitis rose substantially in the 1990s, a fact known to eye institutes in the UK, but contested by the College of Optometrists; and, secondly, that we are on the right track therapeutically with this condition, by using the combination of a biguanide antiseptic and a diamidine, in the form of propamidine isethionate.

There were, however, two major issues in the contribution which we found confusing Firstly, only in 13 of $22(59 \%)$ patients were Koch's postulates ${ }^{2}$ fulfilled and viable Acan thamoeba detected from scrapes of corneal epithelium. The remainder were diagnosed on clinical criteria alone. It is misleading in this context, however, to include responsiveness to the regimen of PHMB or Brolene, or a combination of these, since both compounds have broad spectrum antimicrobial activity. Responsiveness or not to such an agent canno be considered as evidence for the presence or absence of a specific microbe.

Having amassed considerable experience in the cultivation of Acanthamoeba and other free living amoebae ${ }^{3}$ from corneal tissues, which have often been exposed to a cocktail of antimicrobials and other drugs, we have considerable empathy with the authors' difficulties in identifying the amoebae from the standard culture method.

We routinely examine the scrape, unstained and mounted in a buffered saline solution using bright field or phase contrast microscopy. ${ }^{4}$ Our success rate with this method is very high when followed up with culture and histology. This rapid diagnosis permits rational medical therapy to be instituted without delay.

Secondly, one patient in the series became intolerant to the topical drops (PHMB Brolene, or both). The authors state that there were no other toxic effects observed. They do not, unfortunately, provide details of the concentration of PHMB used (one presumes it is calculated as $0.02 \%),{ }^{5}$ its formulation, or the frequency of use of the drugs in their patient group. PHMB is known to have an affinity for anions such as the chloride ions present in a saline solution. PHMB can also interact with various plastics or with non-acid aged glass. These factors may reduce considerably the actual concentration of the compound delivered to the ocular surface. We believe that formulation and storage of this drug is thus of considerable importance when assessing therapeutic response to an Acanthamoeba infection or determining toxicity profiles.

The bisbiguanide chlorhexidine has a similar in vitro acanthamoebicidal effect as PHMB, ${ }^{67}$ although mole for mole the polymeric biguanide appears more so. Physicochemical properties leading to non-specific tissue binding are, however, likely to nullify this concentration effect. ${ }^{8}$ Like PHMB, chlorhexidine has proved efficacy against Acanthamoeba in vivo, albeit in a smaller number of patients. ${ }^{79}$ All of these patients, however, had culture proved Acanthamoeba keratitis which resolved with the combination of chlorhexidine $(0.02 \%)$ in combination with Brolene.
Despite rigorous follow up, no iatrogenic effects were noted. Chlorhexidine salts at concentrations of $0.2 \%$ have been subjected to intensive toxicological investigation by dermatologists and dentists; the compound is also fairly innocuous to the external eye. ${ }^{9}$ It should be noted that no such data are currently available for PHMB. Perhaps for this reason alone, it would be more appropriate to consider chlorhexidine as the first line therapy, possibly in combination with Brolene, for the treatment of Acanthamoeba keratitis. DAVID V SEAL Tennent Institute of Ophthalmology,
Western Infirmary, Glasgow G11 6NT

1 Illingworth CD, Cook SD, Karabatsas CH, Easty outcome. Br f Ophthalmol 1995;79:1078-82.

2 Brock TD. Robert Koch: a life in medicine and bacteriology. Berlin: Springer-Verlag, 1988.

3 Aitken D, Hay J, Kinnear FB, Kirkness CM, Lee WR, Seal DV. Amebic keratitis in a wearer of disposable contact lenses due to a mixed Vahlkampfia and Hartmannella infection. OphVahlkampfia and Ha

4 Hay J, Kinnear F, Kirkness CM, Seal DV Acanthamoeba keratitis: laboratory diagnosis, characterisation of protozoa and treatment Scottish Centre Infect Environ Health 1995;29(95/ 17):90-1.

5 Larkin DFP, Kilvington S, Dart JKG. Treatment of Acanthamoeba keratitis with polyhexamethylene biguanide. Ophthalmology 1992;99:18591.

6 Elder MJ, Kilvington S, Dart JKG. A clinicopathologic study of in vitro sensitivity testing and Acanthamoeba keratitis. Invest Ophthalmo Vis Sci 1994;35:1059-64.

7 Hay J, Kirkness CM, Seal DV, Wright P. Drug resistance and Acanthamoeba keratitis: the quest for alternative antiprotozoal chemotherapy. Eye 1994;8:555-63.

8 Seal DV, Hay J, Kirkness CM. Chlorhexidine or polyhexamethylene biguanide for Acanthamoeba keratitis. Lancet 1995;345:136.

9 Seal D, Hay J, Kirkness CM, Aitken D, Morrell A Booth A, et al. Successful medical therapy of Acanthamoeba keratitis with topical chlorhexidine and propamidine. Eye (in press).

\section{Reply}

EDITOR,-Koch's postulates were intended to be used to prove a causal link between a micro-organism and a disease entity. In the case of Acanthamoeba keratitis a number of other publications, cited in our paper, have previously established this link. This is true of many other infectious diseases where it is considered acceptable in clinical practice to make a firm diagnosis in the absence of positive culture. When considered together, the overal clinical picture of Acanthamoeba keratitis is rather typical: with increasing experience it is frequently possible to make the diagnosis at presentation to a corneal specialty clinic, and start treatment before culture results are available. Positive culture is desirable, but not essential for diagnosis. One of the purposes of our paper was to offer some guidance to clinicians who encounter patients whom they suspect may have Acanthamoeba keratitis. In this context our experience of eyes that have clinical features of the disease but a negative culture is useful, and it would have been misleading to exclude those cases.

In none of our cases did we make a diagnosis based upon response to treatment. Out of a total of 23 eyes, Acanthamoeba was identified by culture of corneal epithelium in 13, by histology in another, and by culture of contact lenses or storage cases (which, in combination with typical clinical features, is sufficient to make the diagnosis) in four. In the five eyes negative for culture and histology, there were clear signs of Acanthamoeba keratitis as detailed in the paper. The rate of positive cul- ture is similar to that of the largest single centre series of cases published to date. ${ }^{1}$ Response to treatment is not in itself a diagnostic criterion, but in the event of failure to respond, reassessment would be called for.

We restricted the discussion of laboratory diagnosis to culture on $E$ coli seeded nonnutrient agar since this is the most widely employed technique and is suitable for use by microbiologists without special expertise in the field. With reference to the use of microscopy for early diagnosis, our own experience has been that it can be difficult to identify unstained amoebae in clinical specimen unless motile trophozoites are clearly seen. An initial diagnosis based upon microscopy should preferably be confirmed by culture. A simple method combining both approaches is to inoculate the sample directly into a $25 \mathrm{~cm}^{3}$ culture flask containing $E$ coli suspended in $10 \mathrm{ml}$ quarter strength Ringer's solution (concentration $3 \times 10^{8} / \mathrm{ml}$ by optical density standards). Amoebae rapidly settle and adhere to the base of the flask, where they can be identified using an inverted phase contrast microscope. The initial impression can be confirmed by observation of multiplication of amoebae after 1 to 3 days of culture in air at $33^{\circ} \mathrm{C}$

The development of intolerance to drops in one patient in our series was probably related to the known epithelial toxicity of propamidine. The formulation of PHMB was identical to that used previously, and was of the same concentration $(0.02 \%){ }^{2}$ The frequency of instillation is given in the methods section.

vs; 1Lastly, treatment with chlorhexidine has been reported only very recently. The use of PHMB was introduced in 1990 in response to the poor results of treatment with propamidine and other compounds. By March 1995 at least 184 eyes had been treated with PHMB in the UK alone (including those in our series). ${ }^{3}$ In these only five treatment failures occurred, and three of these were early cases where PHMB was used late in the disease process after unsuccessful use of propamidine. The use of chlorhexidine was considered during the initial search for an effective alternative to propamidine, and was rejected owing to a lower cysticidal activity than PHMB and concerns about its toxicity. The theoretical merits of both compounds have been debated previously ${ }^{3}$ but it should be noted that in an in vitro model chlorhexidine was toxic to endothelium at a concentration as low as $0.002 \%,{ }^{4}$ while PHMB is relatively non-toxic. ${ }^{5}$ Additionally, chlorhexidine resistance appears to be common in Pseudomonas aeruginosa ${ }^{6}$ and has been reported in the known corneal pathogen Serratia marcescens. ${ }^{7}$ Bacterial co-isolates are commonly reported in Acanthamoeba keratitis, and it has been proposed that Acanthamoeba may gain access to the cornea only after initial ocular infection by bacteria. ${ }^{8}$ It is also possible that secondary bacterial infection might arise in Acanthamoeba keratitis since chronic deepithelialisation is common; this does not appear to occur in patients treated with PHMB. Chlorhexidine may well have a role to play in the treatment of Acanthamoeba keratitis, but the current situation is that while there have now been many reports of the successful use of PHMB in a large number of patients, this is not true of chlorhexidine.

C IILINGWORTH $S$ COOK C KARABUTUS D EASTY 
1 Bacon AS, Frazer DG, Dart JKG, Matheson M, Ficker LA, Wright $P$. A review of 72 consecutive cases of Acanthamoeba keratitis, 1984-1992. Eye 1993;7:719-25.

2 Larkin DF, Kilvington S, Dart JK. Treatment of Acanthamoeba keratitis with polyhexamethylene biguanide. Ophthalmology 1992;99:185-91.

3 Elder MJ, Dart JKG. Chemotherapy for Acanthamoeba keratitis. Lancet 1995;345:791-2.

4 Green K, Livingston V, Bowman K, Hull DS Chlorhexidine effects on corneal epithelium and endothelium. Arch Ophthalmol 1980;98:1273-8.

5 Berry M, Easty DL. Isolated human and rabbit eye: models of corneal toxicity. Toxicology in vitro 1993; $7: 461-4$.

6 Stickler DJ, Thomas B. Antiseptic and antibiotic resistance in Gram-negative bacteria causing urinary tract infection. $\mathcal{F}$ Clin Pathol 1980;33: 288-96.

7 Marrie TJ, Costerton JW. Prolonged survival of Serratia marcescens in chlorhexidine. Appl Environ Microbiol 1981;42:1093-102.

8 Bottone EJ, Madayag RM, Qureshi MN. Acanthamoeba keratitis: synergy between amebic and bacterial cocontaminants in contact lens care systems as a prelude to infection. $f$ Clin systems as a prelude to
Microbiol 1992;30:2447-50.

\section{Fuchs' heterochromic uveitis and sarcoidosis}

EdrToR,--Richard Goble and Philip Murray provoke further interest in the possible cause(s) of Fuchs' heterochromic uveitis (FHU), in reporting five patients with suggested sarcoidosis, including four with raised serum angiotensin converting enzyme (ACE). In patients with sarcoidosis, increased production of ACE is attributed to activated cells within granulomata. In 'granulomatous' uveitis it is reasonable, even in the absence of systemic symptoms, to investigate for the possibility of sarcoidosis. However, it is rarely appropriate to obtain biopsies of ocular tissue for this purpose. We therefore rely on indirect methods of diagnosis.

Weinreb ${ }^{2}$ measured ACE levels in normal controls and found that $4.2 \%$ had significantly raised levels. By comparison, of those with 'granulomatous' uveitis but without evidence of systemic sarcoidosis, $44 \%$ had raised levels. His conclusion, that 'ocular sarcoidosis' may be diagnosed in the absence of systemic evidence, is attractive, though unproved, and would explain a large subset of idiopathic uveitis. However, to extend this group to include forms of uveitis which are untypical of sarcoid related uveitis is mere speculation.

Iris nodules are a recognised feature of FHU, ${ }^{3}$ yet their appearance (small, domeshaped, multiple, and translucent) and position (on the anterior iris surface, mostly peripupillary, scattered symmetrically) differentiate them from nodules seen in granulomatous disease (usually larger, fewer, often irregular in shape, sometimes buried within the stroma). Their presence in FHU cannot per se make a convincing case for a granulomatous aetiology. Indeed, Rothova et $a l,{ }^{4}$ in reporting four black patients with FHU and iris nodules, investigated for sarcoidosis and found no evidence (including normal ACE levels in all four cases). There is no patient with systemic sarcoidosis in the 184 strong Manchester cohort of FHU patients (Jones NP, unpublished data). Also, mutton fat keratic precipitates as described in two of Goble and Murray's patients are not considered to be an accepted part of the FHU spectrum and their appearance should lead one to reconsider the diagnosis.

The evidence for the relevance of raised ACE levels in a small number of patients with possible FHU is therefore unconvincing. The test has significant problems with both specificity and sensitivity, and raised levels are reported in as many as $17 \%{ }^{5}$ in a population of non-sarcoid uveitis. The significance of raised ACE levels in four patients with possible FHU should therefore be statistically justified in the context of the authors' FHU group of patients. Even should this reach statistical significance, the assumption that this represents a form of sarcoidosis is speculative.

NP JONES

Deparment of Ophthalmology, Royal Eye Hospital, Oxford Road, Manchester M13 9WH

1 Goble RR, Murray PI. Fuchs' heterochromic uveitis and sarcoidosis. $\mathrm{Br} f$ Ophthalmol 1995;79:1021-3.

2 Weinreb RN, Kimura SJ. Uveitis associated with sarcoidosis and angiotensin converting enzyme. Am $₹$ Ophthalmol 1980;89:180-5.

3 Jones NP. Fuchs' heterochromic uveitis: a Jones NP. Fuchs heterochromic uveitis: a
reappraisal of the clinical spectrum. Eye 1991;5: reappraisal

4 Rothova A, La Hey E, Baarsma GS, Breebaart AC. Iris nodules in Fuchs' heterochromic uveitis. Am ₹ Ophthalmol 1994;118:338-42.

5 Neves RA, Rodrigues A, Power WJ, PedrosaSeres M, Foster CS. The value of combined serum angiotensin converting enzyme and gallium angiotensin convering enzyme and sis. In: Nus in the diagnosis of ocular sarcoidoRR, Geri I, eds. Advances in ocular immunology. Amsterdam: Elsevier, 1994:353-6.

\section{Reply}

EDTrOR,-We thank Nicholas Jones for his comments on our paper. $\mathrm{He}$ raises a number of important issues including the clinical characteristics of our patients and the difficulty in diagnosing ocular sarcoidosis when biopsy is not possible.

At some time point in their disease each of our five patients showed the characteristic clinical features of FHU. All patients had uniformly distributed, stellate keratic precipitates (KPs), iris stromal atrophy with heterochromia, and no posterior synechiae. Four out of five patients had iris nodules and three had posterior subcapsular cataract. Although mutton fat KPs are not a recognised feature of FHU, very occasionally these patients can develop a supra-added anterior uveitis resulting in the formation of atypical KPs.

Sarcoidosis can be difficult to diagnose particularly in the absence of extraocular features. Although elevated angiotensin converting enzyme (ACE) levels are not specific for sarcoidosis the uveitis in these patients would be highly unlikely to result from any of the other causes of a raised ACE. Also, one patient was Kveim positive and another had chest $x$ ray changes compatible with sarcoidosis.

We agree that a larger series of patients would be required in order to demonstrate any statistically significant association between raised ACE levels and FHU. Nevertheless, the finding of a raised ACE in four patients with a condition that forms only $3 \%$ of all uveitis entities would appear to be more than jus coincidental.

Ocular sarcoidosis may not always present with the typical textbook findings, an example of this would be those patients who have fundal appearance similar to birdshot retinochoroidopathy but are HLA-A29 negative. The features of FHU seen in our patients may be another atypical presentation.

Although FHU has been reported in combination with numerous conditions, a possible association with sarcoidosis has not been previously described. We felt that this was an interesting new finding which would support the theory that FHU may be a secondary phenomenon or a clinical end state of a number of conditions.

RR GOBLE

PI MURRAY

Academic Unit of Ophthalmology, Birmingham and Midland Eye Centre, City Hospital NHS Trust,

Dudley Road,

Birmingham B18 7QH

\section{Determining the importance of eye diseases in Africa}

EDITOR,-In Africa the public health importance of trachoma and xerophthalmia is often underestimated when based on routine surveillance data and even data from population based surveys of low vision and blindness. Surveillance data may under-represent occurrence because both diseases are prevalent in children who rarely complain of it and health personnel seldom examine patients' eyes if they do not have an eye complaint. Population based surveys may under-represent occurrence if cluster sampling is used as this is a weak technique for detecting diseases with focal distribution. These problems are highlighted below using experiences on estimating the importance of these diseases in Ethiopia.

In 1978-80 the Ethiopian Nutrition Institute and the WHO conducted a countrywide assessment on the reporting of xerophthalmia in health centres and hospitals. The study concluded that the condition was rarely recorded. ${ }^{1}$ In the early 1980 s two foci of vitamin A deficiencies were detected in faminefree areas of Arsi, Bale, and Gamu Gofa provinces. ${ }^{2}$ Trachoma was also heavily underreported. In a study of eye conditions at three health centres, where all children under 10 years of age attending the centres for any reason were examined for eye diseases, prevalence of trachoma was 10-fold higher than previously suggested by hospital records.

In 1981 a population based survey of blindness was undertaken. ${ }^{4}$ The survey found a blindness prevalence of between $1.35 \%$ and $1.5 \%$ and trachoma was found to be the major cause. However, the survey failed to detect a high prevalence of xerophthalmia in the country. By chance the xerophthalmia foci were not selected when the random sample of clusters were drawn.

Because of the limitation of the methods discussed above health authorities should identify areas where the population is likely to be at high risk, because of the presence of known risk factors for that condition, and then undertake a sample survey of the children within the high risk areas. This should provide a more realistic insight into the magnitude of the problem in specific high risk areas and direct planning for targeted intervention.

G DE SOLE

1 Pascoe Avenue, BP 6988, Harare, Zimbabwe

1 WHO, UNICEF, USAID, HKI, IVACG. Control of vitamin A deficiency and xerophthalmia. 1982.

2 Lindtiorn B. Xerophthalmia in Gardulla area of southwest Ethiopia. Ethiop Med ₹ 1983;21:16974.

3 De Sole G, Belay Y, Zegeye B. Vitamin A deficiency in southern Ethiopia. Am $\mathcal{f}$ Clin Nutr deficiency in south

4 Budden FH. Blindness in Ethiopia. WHO/STC unpublished report. Geneva: WHO, 1981. 